\title{
User rate and power optimization for HetNets under Poisson cluster process
}

\author{
Xinqi Jiang and Fu-Chun Zheng* (D)
}

\begin{abstract}
Heterogeneous cellular networks (HetNets) consist of different tiers of base stations (BSs) to meet the ever-increasing mobile traffic demand. Due to the random deployment of BSs, Poisson point process (PPP) is often used to model the BS distribution. However, low power small cells are usually clustered around the popular areas, and PPP can not reflect such a feature. To this end, we in this paper consider base station (BS) cooperation and analyze user rate and energy efficiency of HetNets based on a Poisson cluster process (PCP). A calculable formula for the average data rate (or spectral efficiency) and its approximated closed form are derived. Based on this closed form, a power minimization solution with certain spectral efficiency constraint is proposed, and the optimal cooperation radii are derived. Furthermore, we analyze spectral efficiency under a limited number of cooperative BSs in a two-tier network. Finally, we propose a range expansion (RE) scheme and examine the impact of this scheme. The theoretical analyses are verified by simulation results.
\end{abstract}

Keywords: Heterogeneous networks, Poisson cluster process, Energy efficiency, User rate, Stochastic geometry, Range expansion

\section{Introduction}

Comprising macro base stations (BSs) overlaid with dense low power BSs (hence small cells) [1], heterogeneous networks (HetNets) are being deployed to meet the rapid growth in data demand from wireless users, especially those users at the edge of macro cells. Due to the nature of dense deployment for HetNets and the power consumption of all the BSs, energy efficiency (EE) has now been considered as another key performance indicator (KPI) for future wireless networks (e.g., 5G) on top of the traditional KPI of data rate or spectrum efficiency. As a result, not only the data rate but also the EE has recently received enormous attention from the communications community [2-4].

BS locations in dense HetNets tend to be irregular and randomly placed compared with traditional cellular networks, which makes the analysis of the HetNets' performances much more intricate. Fortunately, for the user rate and energy efficiency analysis, some tools from stochastic geometry have proved to be powerful, such as

*Correspondence: fzheng@ieee.org

School of Electronic and Information Engineering, Harbin Institute of

Technology, Shenzhen, China the probability generating functional (PGFL) of Poisson cluster process (PCP), the Palm characterization of PCP, and the Campbell-Mecke theorem. Indeed, these tools have made possible the theoretical analysis of many network metrics, such as average rate, energy consumption, as well as transmission delay.

One major challenge for HetNets is interference management. Coordinated multi-point (CoMP) transmission [5] is an effective scheme to reduce interference by transforming interfering signals to useful signals or to increase space diversity, hence enhancing the performances of HetNets. As such, CoMP is expected to play a key role in future cellular networks as an effective means of meeting higher data rates as well as expanding the indoor and cell-edge coverage. In [6, 7], the authors apply CoMP transmission in HetNets to enhance performances of network, and simulation results illustrate that CoMP can significantly improve the average SE and EE compared to non-CoMP. Thus, a single-stream CoMP transmission scheme (also termed macro-diversity CoMP) is exploited in this paper. 


\subsection{Related work and motivations}

As mentioned above, there has been much effort on applying stochastic geometry to modeling and analyzing HetNets. In most works so far, cellular networks have been modeled as a tractable distribution such as Poisson point process (PPP) [8-10]. In [10], the authors derived the spectral efficiency based on the PPP model and determined the optimal received signal strength (RSS) thresholds under certain approximation. However, most small cells are usually clustered around densely populated areas, and PPP is not always suitable. Investigating a more accurate model therefore becomes imperative. To this end, the Laplace transform of the total interference in HetNets using a PCP was given in [11]. In [12], the authors compared PPP, aggregative point process, and repulsive point process to choose the optimal model for BS deployment in urban areas. In [13], it was stated that the PPP models in some case is more accurate than the hexagonal grid model, but the PCP is able to even more accurately model BS deployment. Assessing KPIs of HetNets based on the PCP model therefore becomes necessary. However, an integrated characterization for aggregate interference as well as the PGFL, based on the PCP network model, has not been reported, although such a characterization is crucial to the theoretical analysis of network performances, such as SE and EE.

Indeed, as both $\mathrm{EE}$ and $\mathrm{SE}$ are now viewed as crucial performance metrics in 5G networks, analysis of EE and SE has gained much attention recently. Reference [14] proposed a new cooperative multiple-input multiple-output (MIMO) scheme to improve the spectrum efficiency while maintaining the same spatial multiplexing and diversity gains as traditional MIMO schemes. Reference [15] studied the EE and area SE with respect to the number and size of microcells. References $[16,17]$ studied the performances of cross-tier cooperation scheme based on the PPP network model, and the numerical results illustrate that such a cooperation strategy can significantly boost ergodic capacity and reduce outage probability. Cross-tier cooperation scheme is expected to be a major technique to provide higher data rates. However, none of the above works has applied this cooperation schemes to a PCP network. As a result, Reference [18] derived new analytical expressions for the coverage probability and area spectral efficiency on clustered device-to-device millimeter wave networks. In [19], the authors investigated the outage probability, the coverage probability, and the average achievable rate based on a tractable lower bound of the PGFL when the nodes follow a PCP. Reference [20] found that the coverage probability in [19] is not always accurate for analyzing PCP network performances. However, the EE and SE of PCP modeled HetNets, based on cross-tier cooperation, have not been analyzed so far.
Over the above situation, this paper targets at the single-stream CoMP over a PCP HetNet, where cross-tier cooperation is based on the RSS threshold. From the perspectives of EE and SE, we derived a tractable result for SE based on PCP and then proposed an energy optimization solution. Moreover, we also analyzed spectral efficiency in case of the limited number of cooperative BSs in a twotier HetNet and proposed a range expansion (RE) scheme based on CoMP, which balances the load of macro BS.

\subsection{Contributions}

The main contributions of this paper are therefore as follows: (1) a computationally efficient formula for spectral efficiency is derived under the PCP model, and then approximated as a closed form; (2) a comprehensive comparison with the corresponding PPP results is carried out; (3) a power optimization scheme is proposed by optimizing the cooperative radius of each tier; and (4) a scheme based on a limited number of cooperative BSs and a range expansion (RE) strategy are proposed, which can ease the load of macro BS and enhance the network EE.

\subsection{Paper organization}

The remainder of this paper is structured as follows. In Section 3, we give a brief description on a particular PCP model and present the downlink system model. The formula of spectral efficiency is derived based on PCP and an optimal solution for each tier's cooperative radius is determined in Section 4. In Section 5, we propose an effective BS cooperation and RE strategy based on CoMP. Section 6 presents the numerical results and related illustrations. A list of major symbols and their meanings are shown in Table 1.

\section{Methods/experimental}

The methods and analysis in this paper are based on stochastic geometry and are described in Section 3, while the verification is based on Monte Carlo simulations as presented in Section 6. This paper contains no experiments.

\section{PCP and downlink system model}

\subsection{Poisson cluster process (PCP)}

A Poisson cluster process (PCP) consists of two kinds of point processes: the parent points in each tier following homogeneous Poisson point process (PPP) with the density $\lambda_{p, k}$ form the centers of the clusters, while the daughter nodes are scattered around cluster centers. Matern cluster process (MCP) is a special case of PCP, in which the daughter nodes are of uniform distribution within a circle of radius $R_{a}$ with the corresponding parent point being the cluster center. The parent points indicate the centers of hotspots, and the daughter nodes represent the locations of BSs. In this paper, we mean MCP 
by PCP. This special PCP model and the PPP model are illustrated in Fig. 1. In each cluster of the $k$ th tier, the number of daughter nodes is fixed to be $c_{k}$, the mean density of the $k$ th tier is therefore $c_{k} \lambda_{p, k}$. The probability density function of such PCP daughter nodes is given by

$$
f(d)=\left\{\begin{array}{l}
\frac{1}{\pi R_{a}^{2}} \quad\|d\| \leq R_{a} \\
0 \quad \text { otherwise }
\end{array}\right.
$$

where $d$ is a daughter node's position relative to the cluster center, and $\|d\|$ is the distance between the daughter node and the center of the relevant cluster.

\subsection{System model}

We consider a general model of downlink HetNets which includes $K$ network tiers, and the BS positions of each tier form a PCP denoted by $\Phi$ on $\mathbb{R}^{2}$. BSs across tiers are distinguished by their deployment mean density $c_{k} \lambda_{p, k}$, transmit power $p_{k}$, and path loss exponents $\alpha_{k}$. User density is denoted by $\lambda_{u}$. Both BSs and users are equipped with a single antenna. Without any loss of generality, the general path loss is given by $P L(x)=\|x\|^{-\alpha_{k}}$, where $\|x\|$ indicates the distance between a typical user at the origin and BS at $x$. Inspired by [10], we also consider a user-centric cross-tier BS collaboration scenario. Figure 2 illustrates a two-tier HetNet involving a mix of macro and pico BSs, where the typical user can be served by cooperative BSs from both tiers.

Table 1 Major symbols used in this paper

\begin{tabular}{|c|c|c|}
\hline Variable & Meaning & Unit \\
\hline$\lambda_{u}$ & User density & $m^{-2}$ \\
\hline$\lambda_{p, k}$ & Parent points density of the $k$ th tier & $m^{-2}$ \\
\hline \multirow[t]{2}{*}{$c_{k}$} & Number of daughter points within each & None \\
\hline & cluster of the $k$ th tier & \\
\hline$R_{a, k}$ & Cluster radii of the $k$ th tier & $m$ \\
\hline$R_{k}$ & Cooperative radii of the $k$ th tier & $m$ \\
\hline$R_{0, k}$ & Optimal cooperative radii of the $k$ th tier & $m$ \\
\hline$P_{u}$ & Average power consumption on serving one user & W \\
\hline$p_{k}$ & Transmit power of the $k$ th tier & W \\
\hline \multirow[t]{2}{*}{$P_{b h, k}$} & Backhaul power dissipation of the $k$ th & W \\
\hline & tier on serving one user & \\
\hline \multirow[t]{2}{*}{$P_{s p, k}$} & Signal processing power consumption of the & W \\
\hline & kth tier on serving one user & \\
\hline$\theta_{k}$ & RSS threshold of the $k$ th tier & W \\
\hline$a_{k}$ & Slope of the kth tier power consumption & None \\
\hline$U_{k}$ & Number of cooperative BSs in the $k$ th tier & None \\
\hline$\alpha_{k}$ & Path loss exponents of the $k$ th tier & None \\
\hline$h_{k}$ & Power fading coefficient of the $k$ th tier & None \\
\hline
\end{tabular}

We employ the Palm measure [21] to characterize the total signal and the aggregate interference at the typical user located at the origin and served by cross-tier cooperative BSs. For convenience, the set of cooperative BSs is denoted by $C_{C}$. BSs in the $C_{C}$ transmit the same data to the typical user (hence a non-coherent macro-diversity CoMP strategy) and the RSS at the user exceeds some RSS threshold. Namely, for the $k$ th tier, the BS located at $x_{k}$ belongs to the cooperative group $C_{C}$ if $p_{k} h_{k}\left\|x_{k}\right\|^{-\alpha_{k}} \geq \theta_{k}$, where $\theta_{k}$ is the RSS threshold for the $k$ th tier, and $h_{k}$ denotes the power fading coefficient of the $k$ th tier.

Assuming that the cooperative region in each tier is circular, the cooperative radius $R_{k}$ can then be given by

$$
R_{k}=\left(\frac{p_{k} h_{k}}{\theta_{k}}\right)^{1 / \alpha_{k}}
$$

Denoting by $C_{I}=\Phi \backslash C_{C}$ the set of the interfering BSs, the signal-to-interference-plus-noise ratio (SINR) at the typical user is given by

$$
\operatorname{SINR}=\frac{\sum_{x_{k} \in C_{C}} p_{k} h_{k}\left\|x_{k}\right\|^{-\alpha_{k}}}{\sum_{x_{k} \in C_{I}} p_{k} h_{k}\left\|x_{k}\right\|^{-\alpha_{k}}+\sigma^{2}},
$$

where $\sigma^{2}$ is the noise power.

\section{Spectral efficiency and power optimization}

In this section, we derive and optimize two key metrics, i.e., the spectral efficiency (SE) and energy efficiency (EE) based on the probability generating functional (PGFL) of the Poisson cluster process in a $K$-tier HetNet. Both metrics depend on the SINR in [3]. As in [10], the Laplace transform is used to characterize the aggregate interference (the denominator) and the total signal power (the numerator plus the denominator). For comparison, the same denotations have also been employed as in [10].

\subsection{Laplace transform of the total signal power}

For the proposed PCP model, the number of daughter nodes in each cluster is assumed to be fixed, and the parent points are not included. All cooperative BSs are assumed to transmit the same signal to the "typical user" at the origin. The total signal power (the signal plus the interference) received at this user is given by

$$
P=\sum_{x \in \Phi} p_{k} h_{k}\|x\|^{-\alpha_{k}}=\sum_{k=1}^{K} \sum_{x_{k} \in \phi_{k}} p_{k} h_{k}\left\|x_{k}\right\|^{-\alpha_{k}}
$$

where $\phi_{k}$ denotes the set of BS at the $k$ th tier.

From the above formula, the Laplace transform of total signal power $P$ is 


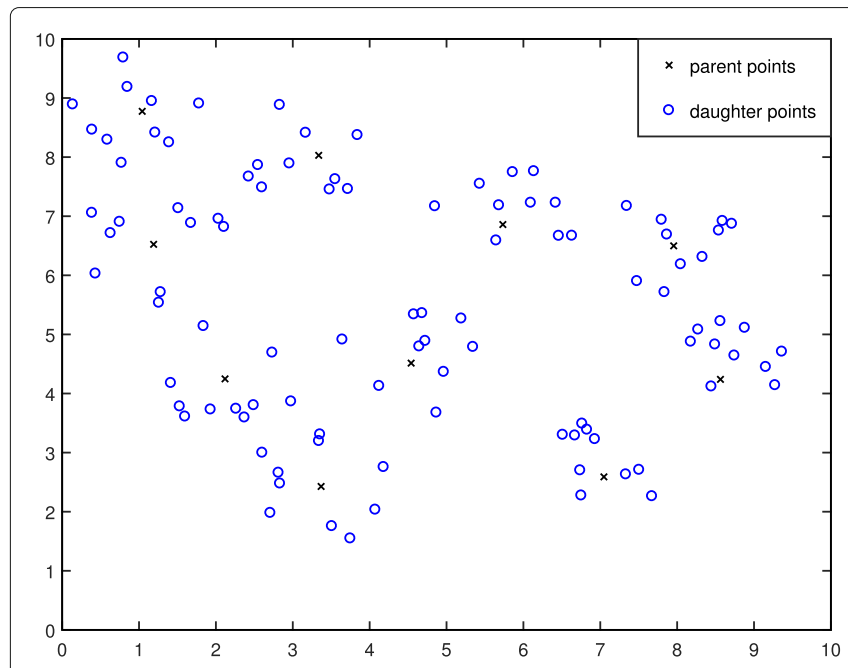

(a)

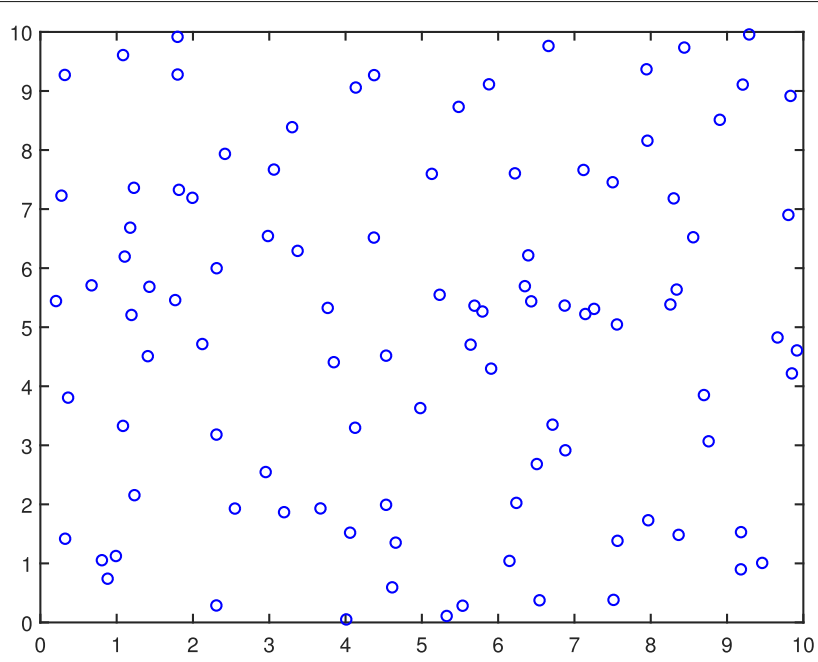

(b)

Fig. 1 The PCP model and PPP model with the same average intensity $\bar{\lambda}=1$ for comparison. a Poisson cluster process $\left(\lambda_{p}=0.1, \bar{c}=10\right)$. b Poisson point process $(\bar{\lambda}=1)$

$$
\begin{aligned}
\mathcal{L}_{P}(s) & =E_{P}[\exp (-s P)] \\
& =\prod_{k=1}^{K} E_{h_{k}, x_{k}}\left[\exp \left(-s \sum_{x_{k} \in \phi_{k}} p_{k} h_{k}\left\|x_{k}\right\|^{-\alpha_{k}}\right)\right] \\
& =\prod_{k=1}^{K} E\left[\prod_{x_{k} \in \phi_{k}} \mathcal{L}_{h}\left(s p_{k}\left\|x_{k}\right\|^{-\alpha_{k}}\right)\right] \\
& \stackrel{(a)}{=} \prod_{k=1}^{K} E\left[\prod_{x_{k} \in \phi_{k}} \frac{1}{1+s p_{k}\left\|x_{k}\right\|^{-\alpha_{k}}}\right],
\end{aligned}
$$

where $\mathcal{L}_{h}\left(s p_{k}\left\|x_{k}\right\|^{-\alpha_{k}}\right)$ denotes the Laplace transform of received signal power at $x_{k}$, and (a) is due to the fact that $h_{k}$ is exponentially distributed with mean one.

On the other hand, the above expression is directly related to the PGFL of PCP as given in [22]

$$
\tilde{G}(v)=E\left[\prod_{x \in \phi} v(x)\right]=\exp \left\{-\lambda_{p} \int_{\mathbb{R}^{2}}\left[1-\beta(y)^{c}\right] d y\right\},
$$

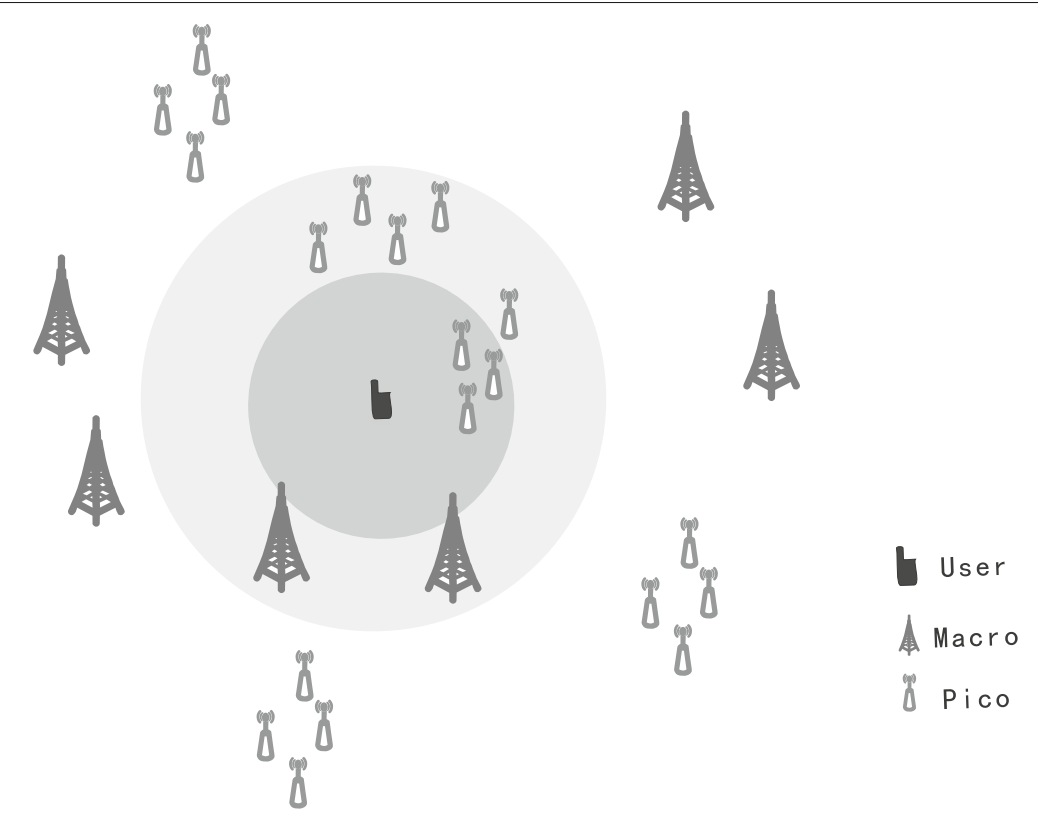

Fig. 2 A typical user is served by macro and pico BSs, where a small circle and a large circle denote pico BSs and macro BSs cooperative areas, respectively 
where

$$
\beta(y)=\int_{\mathbb{R}^{2}} v(x-y) f(x) d x,
$$

and $c$ denotes the number of daughter nodes in each cluster.

The lower bounded PGFL and the upper bounded conditional PGFL of the PCP are given by [19]. In order to evaluate accurately the spectral efficiency, we derive below the precise expression by algebraic operations.

Lemma 1 The Laplace transform (i.e., (5)) of total signal power $P$ is estimated as

$$
\mathcal{L}_{P}(s)=\prod_{k=1}^{K} \exp \left\{-\lambda_{p, k} 2 \pi \int_{0}^{\infty}\left[1-B_{k}(r)^{c_{k}}\right] r d r\right\}
$$

where

$$
B_{k}(r)=\frac{1}{1+s p_{k}\|r\|^{-\alpha_{k}}}
$$

Proof Let $v(x)=\frac{1}{1+s p_{k}\|x\|^{-\alpha_{k}}}$. By applying (6) to (5), we have

$$
\begin{aligned}
& \int_{\mathbb{R}^{2}}\left[1-\beta_{k}(y)^{c_{k}}\right] d y \\
& =\int_{\mathbb{R}^{2}}\left(1-\left(\int_{\mathbb{R}^{2}} \frac{f(x)}{1+s p_{k}\|x-y\|^{-\alpha_{k}}} d x\right)^{c_{k}}\right) d y \\
& \stackrel{(a)}{=} \int_{\mathbb{R}^{2}}\left(1-\left(\int_{\mathbb{R}^{2}} \frac{f(x)}{1+s p_{k}\|r\|^{-\alpha_{k}}} d x\right)^{c_{k}}\right) d r \\
& =\int_{\mathbb{R}^{2}}\left(1-\left(\frac{1}{1+s p_{k}\|r\|^{-\alpha_{k}}}\right)^{c_{k}}\right) d r \\
& \stackrel{(b)}{=} 2 \pi \int_{0}^{\infty}\left[1-B_{k}(r)^{c_{k}}\right] r d r,
\end{aligned}
$$

where (a) uses the change of variables $\|r\|=\|x-y\|$, and (b) follows from the polar representation.

Then by substituting (8) into (6) and then (5), we obtain (7) in Lemma 1. Computing the integral in (6) numerically is a very arduous task, but (7) in Lemma 1 is now much more computationally efficient.

\subsection{Spectral efficiency}

From the information theory, we can achieve Shannon bound $\ln (1+$ SINR) for an instantaneous SINR. Thus, the spectral efficiency in units of nats $/ \mathrm{s} / \mathrm{Hz}$ is calculated as

$$
\begin{aligned}
\tau & =E_{\text {SINR }}[\ln (1+\mathrm{SINR})] \\
& =E_{h_{k}, x_{k}}\left[\ln \left(1+\frac{\sum_{x_{k} \in C_{C}} p_{k} h_{k}\left\|x_{k}\right\|^{-\alpha_{k}}}{\sum_{x_{k} \in C_{I}} p_{k} h_{k}\left\|x_{k}\right\|^{-\alpha_{k}}+\sigma^{2}}\right)\right] \\
& =E_{P, I}\left[\ln \left(\frac{P+\sigma^{2}}{I+\sigma^{2}}\right)\right],
\end{aligned}
$$

where $P$ and $I$ denote the total signal power and aggregate interference power, respectively.

Theorem 1 In a K-tier HetNet, the spectral efficiency of a typical user at the origin under the proposed model can be evaluated as

$$
\begin{array}{r}
\tau=\int_{0}^{\infty}\left[\prod_{k=1}^{K} \exp \left\{\lambda_{p, k} 2 \pi \int_{R_{k}}^{\infty}\left[B_{k}(y)^{c_{k}}-1\right] y d y\right\}-\right. \\
\left.\prod_{k=1}^{K} \exp \left\{\lambda_{p, k} 2 \pi \int_{0}^{\infty}\left[B_{k}(y)^{c_{k}}-1\right] y d y\right\}\right] \frac{e^{-s \sigma^{2}}}{s} d s .
\end{array}
$$

\section{Proof Please see Appendix 1.}

Although it is not a closed form, the above integral can be analysed numerically. As $B_{k}(y)^{c_{k}}-1<0$, the spectral efficiency is an increasing function of the cooperative radii $R_{k}$. As such, we can design a larger cooperative region of BS in HetNets to meet higher data rates (subject to the overhead incurred).

In order to compare the performances (i.e., SE and EE) under PPP and PCP, we adopt the same setup for a typical user as in a PPP model. Assuming fading coefficient $h \sim \exp (1)$, the spectral efficiency under PPP [10] with the density $\lambda_{p p p, k}=c_{k} \lambda_{p, k}=\lambda_{k}$ can be express as

$$
\begin{aligned}
\tau_{p p p}=\int_{0}^{\infty} \frac{e^{-s \sigma^{2}}}{s}\left\{\exp \left[-\sum_{k=1}^{K} \pi \lambda_{k} B\left(R_{k}, s p_{k}\right)\right]-\right. \\
\left.\exp \left[-\sum_{k=1}^{K} \pi \lambda_{k} B\left(0, s p_{k}\right)\right]\right\} d s,
\end{aligned}
$$

where

$$
\begin{aligned}
& B\left(R_{k}, s p_{k}\right)=\int_{R_{k}^{2}}^{\infty} \frac{s p_{k}}{u^{\frac{\alpha_{k}}{2}}+s p_{k}} d u, \\
& B\left(0, s p_{k}\right)=\left(s p_{k}\right)^{\frac{2}{\alpha_{k}}} \Gamma\left(1+\frac{2}{\alpha_{k}}\right) \Gamma\left(1-\frac{2}{\alpha_{k}}\right),
\end{aligned}
$$

and $\Gamma(\cdot)$ denotes the gamma function.

Due to the complexity of the spectral efficiency expression in (10), we now derive a closed form by ignoring 
the noise (interference is the dominated issue in a dense HetNet).

Lemma 2 Ignoring the noise in dense HetNets, Eq. (10) can be simplified as follows

$$
\tau \stackrel{(a)}{\approx} q-\ln f
$$

where

$$
\begin{aligned}
& q=\int_{0}^{\infty}\left[e^{-s}-\prod_{k=1}^{K} \exp \left(2 \pi \lambda_{p, k} \int_{0}^{\infty}\left[B_{k}(y)^{c_{k}}-1\right] y d y\right)\right] \frac{d s}{s}, \\
& f=\sum_{k=1}^{K} \pi c_{k} \lambda_{p, k} p_{k} \frac{2}{\alpha_{k}-2} R_{k}^{2-\alpha_{k}} .
\end{aligned}
$$

\section{Proof Please see Appendix 2.}

Note that $q$ is not related to the cooperative radii, and it is a constant for a given deployment density and transmit power. As shown in Appendix 2, $q-\ln (f)$ is in fact the lower bound of $\tau$ in (10). When $R_{k}$ becomes larger, however, the gap between (10) and $q-\ln (f)$ rapidly tapers off, hence the close approximation of (12) for $\tau$. Equation (12) is a closed formula, which makes the following optimization problem easier to solve.

\subsection{Minimizing energy consumption via optimizing cooperative radii}

For the proposed model, the BSs inside the cooperative clusters can communicate with the typical user located at the origin, and the average power consumption when serving such a user is given by [10]

$$
\begin{array}{r}
P_{u}=\sum_{k=1}^{K}\left[\pi R_{k}^{2} c_{k} \lambda_{p, k}\left(P_{b h, k}+P_{s p, k}+a_{k} \cdot p_{k}\right)+\right. \\
\left.\frac{c_{k} \lambda_{p, k}}{\lambda_{u}} P_{0, k}\right],
\end{array}
$$

where $P_{b h, k}$ is the backhaul power dissipation when serving one user (i.e., the typical user) in the $k$ th tier, $P_{s p, k}$ denotes the corresponding signal processing power consumption, and $a_{k}$ denotes the slope of the $k$ th tier power consumption with respect to transmit power $p_{k}$. The second term of $P_{u}$ in (13) denotes the average static power for serving one user, and it is independent of the load of BSs.

In the following, we will provide a solution for optimizing the average power consumption of the HetNet for the typical user in (13). In reality, it is very important to determine the network parameters which can minimize the network power consumption. Since the average energy consumption $P_{u}$ per user and the average consumed energy $P_{a v}$ are related with $P_{a v}=\lambda_{u} \times P_{u}$, minimizing $P_{u}$ is critical to designing an energy-saving network.

For a given cooperative radius $R_{1}$ of the first tier, we want to find out the optimal cooperative radius $R_{2}$ of the second tier in a two-tier HetNet, which minimizes the energy consumption while ensuring a certain rate to the typical user. Note that the energy consumption in (13) increases with the cooperative radii, which suggests that we should determine the minimum cooperative radii while guaranteeing the minimum user rate. Intuitively, the user rate should increase with cooperative areas, and the rate in (10) is indeed an increasing function of the cooperative radii. Assuming that the first-tier cooperative radius $R_{1}$ is known, we can determine the optimal value of the second-tier cooperative radius $R_{2}$ through dichotomy. The problem will be transformed into a simple problem with a single variable (with the constraint from (10)):

$$
\begin{aligned}
& \min R_{2} \\
& \text { s.t. } \int_{0}^{\infty}\left[\prod_{k=1}^{2} \exp \left\{\lambda_{p, k} 2 \pi \int_{R_{k}}^{\infty}\left[B_{k}(y)^{c_{k}}-1\right] y d y\right\}-\right. \\
& \left.\prod_{k=1}^{2} \exp \left\{\lambda_{p, k} 2 \pi \int_{0}^{\infty}\left[B_{k}(y)^{c_{k}}-1\right] y d y\right\}\right] \frac{e^{-s \sigma^{2}}}{s} d s \geq \tau_{0}
\end{aligned}
$$

The above problem formulation can be solved by the well-known dichotomy algorithm ${ }^{1}$.

Now, we want to form the general problem to determine the optimal values for $R_{1}, R_{2}, \cdots, R_{K}$ in a $K$-tier network, which minimize the power consumption in (13) while satisfying the minimum spectral efficiency requirement. Note that the second term of $P_{u}$ in (13) is independent of $R_{1}, R_{2}, \cdots, R_{K}$, and can be ignored. Hence, by applying (12) and (13), the optimization problem can be formulated as follows:

$$
\begin{aligned}
\min _{\left\{R_{1}, R_{2}, \cdots, R_{K}\right\}} P_{u 1} & =\sum_{k=1}^{K} \pi R_{k}^{2} \lambda_{p, k} c_{k}\left(P_{b h, k}+P_{s p, k}+a_{k} p_{k}\right) \\
\text { s.t. } q-\ln f & =\tau_{0}
\end{aligned}
$$

Clearly, both the constraint condition and the optimization objective function are of closed form. The problem can then be solved easily by a linear programming method as follows.

Theorem 2 The optimal cooperative radii approximately satisfy

\footnotetext{
${ }^{1}$ The dichotomy algorithm (also commonly known as binary search algorithm) means, in our case, the following operation: select a very large $R_{2}$ value at the beginning and then test the constraint in (14) at the middle point of $R_{2}$ (i.e., $0.5 R_{2}$ ). If (14) is satisfied, take the middle point of the lower half of $R_{2}$ and test the constraint in (14) again; if not, take the middle point of the upper half of $R_{2}$ and test (14). Repeat the same steps until the process converges to a certain value (which is the optimal vale for $R_{2}$ ).
} 


$$
\begin{aligned}
& R_{o, k}=\left(\frac{p_{k} P_{a, i}}{p_{i} P_{a, k}} R_{o, i}^{\alpha_{i}}\right)^{\frac{1}{\alpha_{k}}} \\
& f=\sum_{k=1}^{K} \pi c_{k} \lambda_{p, k} p_{k} \frac{2}{\alpha_{k}-2} R_{o, k}^{2-\alpha_{k}}=\exp \left(q-\tau_{0}\right)
\end{aligned}
$$

where $R_{o, k}$ denotes the optimal value of the $k$ th tier cooperative radius, and $P_{a, i}=P_{b h, i}+P_{s p, i}+a_{i} p_{i}$ denotes the total power dissipation when serving a typical user in the $i$ th tier.

\section{Proof Please refer to Appendix 3.}

Combining (16) and (17), the optimal value of each tier can be determined by solving the resultant system of nonlinear equations for a given data rate $\tau_{0}$, which optimizes the PCP networks' energy consumption.

Once the power $P_{u}$ has been minimized, the network energy efficiency $\eta_{e e}$, defined as the ratio of the average spectral efficiency to the average power consumption, can be calculated as

$$
\eta_{e e}=\frac{\tau}{P_{u}}
$$

\section{Limited BS number and range expansion}

In this section, we examine two implementation variations of the above collaboration strategy: (a) limited number of cooperative BSs and (b) range expansion (RE).

\subsection{Limited number of cooperative BSs}

In order to accommodate the ever-increasing traffic demand, low power BSs are densely deployed in HetNets. The number of cross-tier cooperative BSs for each user may be too high in popular areas, due to the overhead incurred and the marginal rate increase.

As a result, the number of associated BSs at a typical user should be limited to an acceptable level. Therefore, we now propose a cooperation tactic where the number of cooperative BSs is restricted to $N(N \leq 4)$. In other words, the typical user at the origin communicates with $N$ BSs based on maximum RSS, and all other BSs become noncooperative. Of course, the SE always increases when a new $\mathrm{BS}$ is added to the cooperative BS set, but the SE gain becomes more and more marginal and the overhead still increases linearly. Hence, there exists a tradeoff between $\mathrm{SE}$ and the overhead (including designing costs of BS and energy consumption). It is therefore meaningful to put a limit on the number of cooperative BSs, which can optimize EE and the overhead cost efficiency. As an example, we consider a two-tier HetNet, and the cooperative BSs number in this case is given by

$$
\sum_{k=1}^{2} U_{k}=N \quad(N \leq 4)
$$

where $U_{k}$ is the number of cooperative BSs in the $k$ th tier.

The effect of this BS constraint will be shown via simulation.

\subsection{Range expansion}

In HetNets, deployment of macro BSs are for large coverage ranges, which may lead to a heavy load for macro BSs in crowded areas, and the energy consumption also heavily depends on the loading of macro BSs due to macro BSs' high power consumption. Therefore, we now propose a range expansion (RE) scheme based on CoMP to alleviate the loading of macro BSs and improve the EE.

Generally, the transmission power of macro BSs is much larger than that of pico BSs, and as a result, many users will still receive the strongest signal from the macro BS even in hotspots. The RE scheme is to favor the selection of low power BSs by introducing a bias to the association threshold for low power BSs [23]. Due to the smaller coverage, lower power BSs can provide more resource blocks to each user than macro BSs. References [23-25] have shown that RE can achieve load balancing and enhance performances of networks without a loss of average user throughput. For example, in a two-tier HetNet, due to the bias, users are more likely to connect to the pico BSs than the macro BSs even if $p_{1} h_{1} r_{1}^{-\alpha_{1}}>p_{2} h_{2} r_{2}^{-\alpha_{2}}$. Figure 3 illustrates how the typical user is served based on the RE scheme in such an example. Here, we consider a two-tier HetNet comprising macro BSs and pico BSs, which follow a PCP. Consequently, the macro BSs can collaborate to serve the typical user at the origin only when $p_{1} h_{1}\|x\|^{-\alpha_{1}} \geq \varepsilon_{1} \theta^{*}$ and the pico BSs can serve this user if $\varepsilon_{2} p_{2} h_{2}\|x\|^{-\alpha_{2}} \geq \theta^{*}$, where $\theta^{*}$ denotes normalized RSS threshold (each tier then still has the same RSS threshold), $\varepsilon_{1}$ and $\varepsilon_{2}$ are the first power bias and the second power bias, respectively. Therefore, the cooperative radii after biasing are given by

$$
\begin{aligned}
& R_{1}^{*}=\left(\frac{p_{1} E\left[h_{1}\right]}{\varepsilon_{1} \theta^{*}}\right)^{1 / \alpha_{1}} \\
& R_{2}^{*}=\left(\frac{\varepsilon_{2} p_{2} E\left[h_{2}\right]}{\theta^{*}}\right)^{1 / \alpha_{2}}
\end{aligned}
$$

where $R_{1}^{*}$ and $R_{2}^{*}$ denote the modified macro-tier radius and modified pico-tier radius after utilizing a power bias, respectively.

From above expressions, the macro BSs cooperation region shrinks but the pico BSs cooperation region expands when the power bias coefficient is greater than 1 . The energy efficiency under various bias values will be examined via simulation in the next section. 


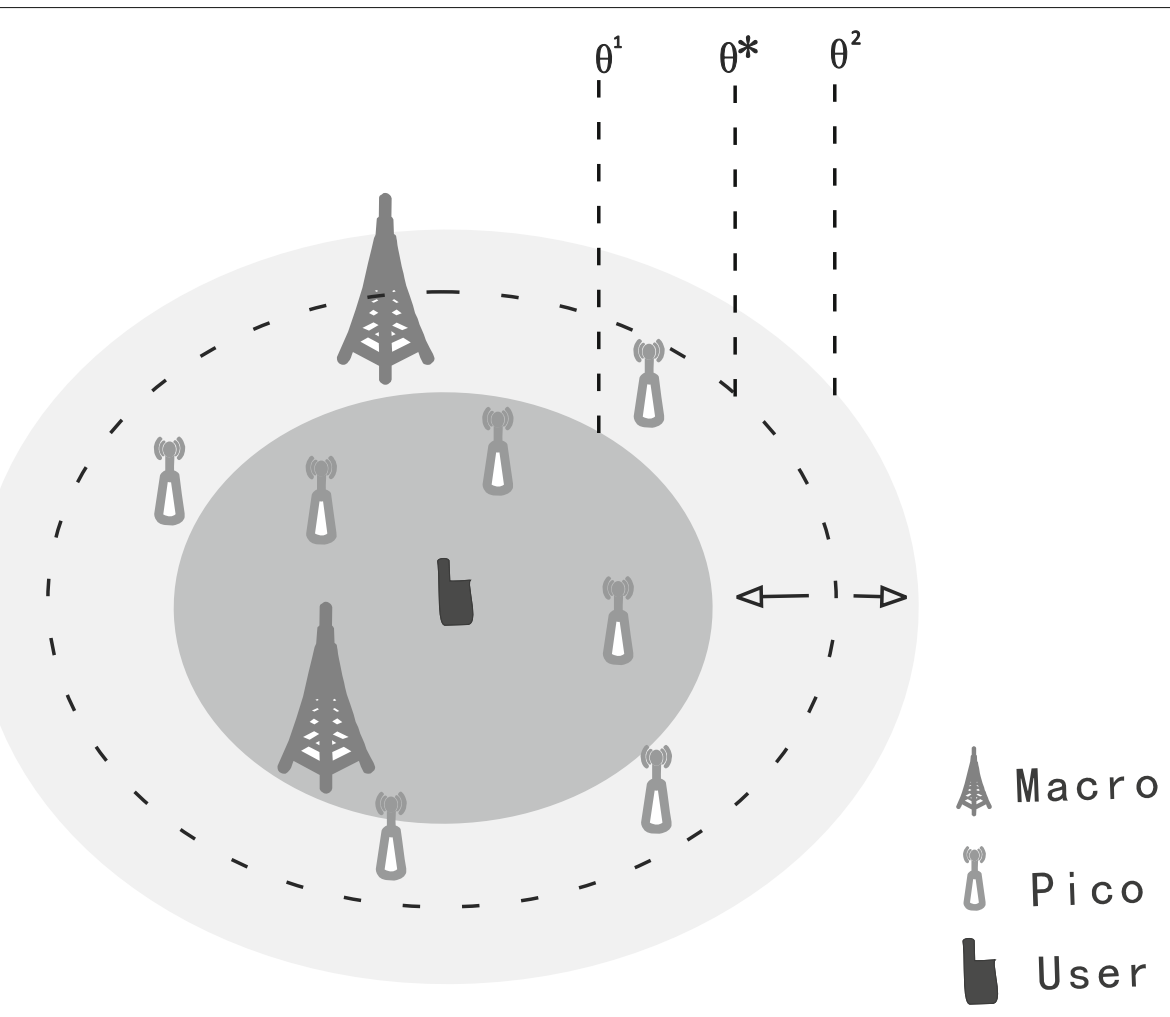

Fig. 3 The typical user is served by macro and pico BSs, where the dashed circle denotes the normalized RSS threshold $\theta^{*}$, while $\theta^{1}$ and $\theta^{2}$ denote the macro-tier and pico-tier RRS thresholds, respectively

\section{Numerical results and discussions}

In this section, we present some numerical results of the user rate and energy efficiency for a two-tier HetNet consisting of macro and pico BSs. The PCP cluster radii of this two-tier HetNet are $R_{a, 1}=200 \mathrm{~m}$ and $R_{a, 2}=100 \mathrm{~m}$, respectively. Unless otherwise specified, the parameters are those from [26]: the transmit power $p_{1}=20 \mathrm{~W}, p_{2}=$ $0.13 \mathrm{~W}$, and the slope of the power consumption: $a_{1}=$ $4.7, a_{2}=4$. We now compare the results under the same parameters for two different point processes: PCP and PPP.

\subsection{Validation of Laplace transform on PCP model}

Figure 4 shows simulation and numerical results of the Laplace transform for a two-tier HetNet computed (i.e., (7)) under the PCP model. The PCP parameters are $\lambda_{p, 1}=1 /\left(\pi \times 300^{2}\right), \lambda_{p, 2}=1 /\left(\pi \times 200^{2}\right), p_{1}=40 \mathrm{~W}, p_{2}=$ $0.13 \mathrm{~W}, c_{1}=1, c_{2}=10$, and the cooperative radii $R_{1}=$ $400 \mathrm{~m}, R_{2}=100 \mathrm{~m}$. The simulation results match well with the analytical integrations in the whole range of $s$ and different path loss exponents (assuming $\alpha_{k}=\alpha$ here), which verifies the validity of the derived Laplace transform of the aggregate interference and total signal powers. The reason for the differences among the $\alpha$ curves is that for larger a $\alpha$ value, the total interference attenuates more, while the Laplace transform is an exponentially decaying function.

\subsection{Comparison between PPP and PCP}

Figure 5 compares the spectral efficiency (SE) versus the first-tier and the second-tier cooperative radii for PPP and PCP models. In order to compare PCP and PPP, we have chosen some parameters as in [10]: $\lambda_{1}=1 /(\pi \times$ $\left.250^{2}\right), \lambda_{2}=1 /\left(\pi \times 50^{2}\right), \alpha_{1}=4.3, \alpha_{2}=3.8$, and set $c_{1}=1, c_{2}=4$. As can be seen from Fig. 5, the average user rate of PCP is worse than that of PPP, and the reason is that the main interferers are more likely to be closer to the typical user for PCP network (hence the higher interference level) than in the case of PPP. Furthermore, the spectral efficiency increases with the macro-tier cooperative radius and the pico cooperative radius, and this is due to the number of cooperative BSs increasing for larger cooperative regions. We note that the pico-tier BSs after some sufficiently large distance contribute very marginally to the user rate. Therefore, the RSS threshold of the second tier should not be too low (to limit the number of the second-tier cooperative BSs), considering the corresponding collaboration overhead.

Figure 6 shows the energy efficiency (EE) versus the mean network cooperative radii $R_{1}$ and $R_{2}$ for PCP and PPP. The network model parameters are $\lambda_{u}=1 /(\pi \times$ $\left.50^{2}\right), c_{1}=1, c_{2}=4, P_{b h, 1}=P_{b h, 2}=11 \mathrm{~W}, P_{s p, 1}=$ $55 \mathrm{~W}, P_{s p, 2}=2.5 \mathrm{~W}, P_{0,1}=75 \mathrm{~W}$, and $P_{0,2}=4.3 \mathrm{~W}$. We can see that the pico-tier cooperative radius $R_{2}$ 


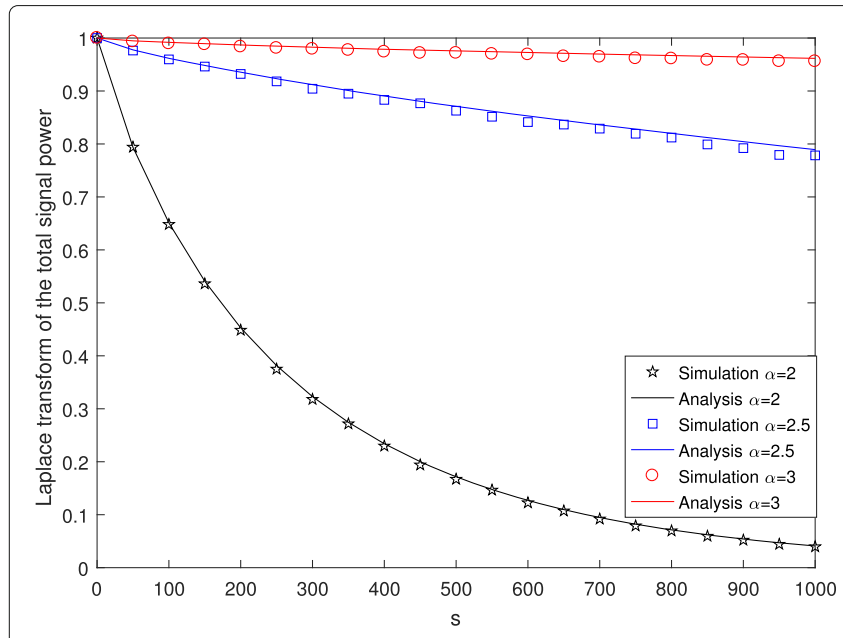

(a)

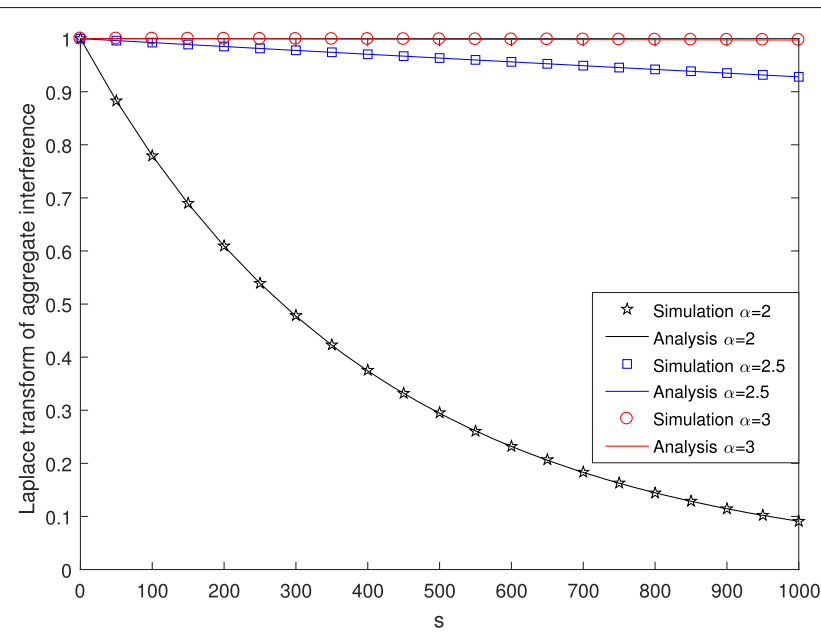

(b)

Fig. 4 The Laplace transform for PCP with different $\alpha$. $\mathbf{a}$ The Laplace transform of the total signal power. $\mathbf{b}$ The Laplace transform of the aggregate interference

has an optimal value for a given $R_{1}$ which leads to a maximum EE. The case is similar to that of PPP, but the EE of PCP is poorer compared with that of PPP, since the average rate of PPP is better than that of PCP, and energy consumption of the two cases are the same due to the same mean network density. Note that the EE is a decreasing function of the macro-tier cooperative radius, and this is because a large cooperative area of a macro-tier results in an increase in the number of the cooperative macro BSs, while the main energy consumption is heavily dependent on the number of cooperative macro BSs.

\subsection{Validity of Lemma 2}

Figure 7 shows that the optimal and the upper bound cooperative radius values of the second tier versus the minimum user rate for two different cooperative radii of the first tier . By utilizing (12), we can acquire the upper

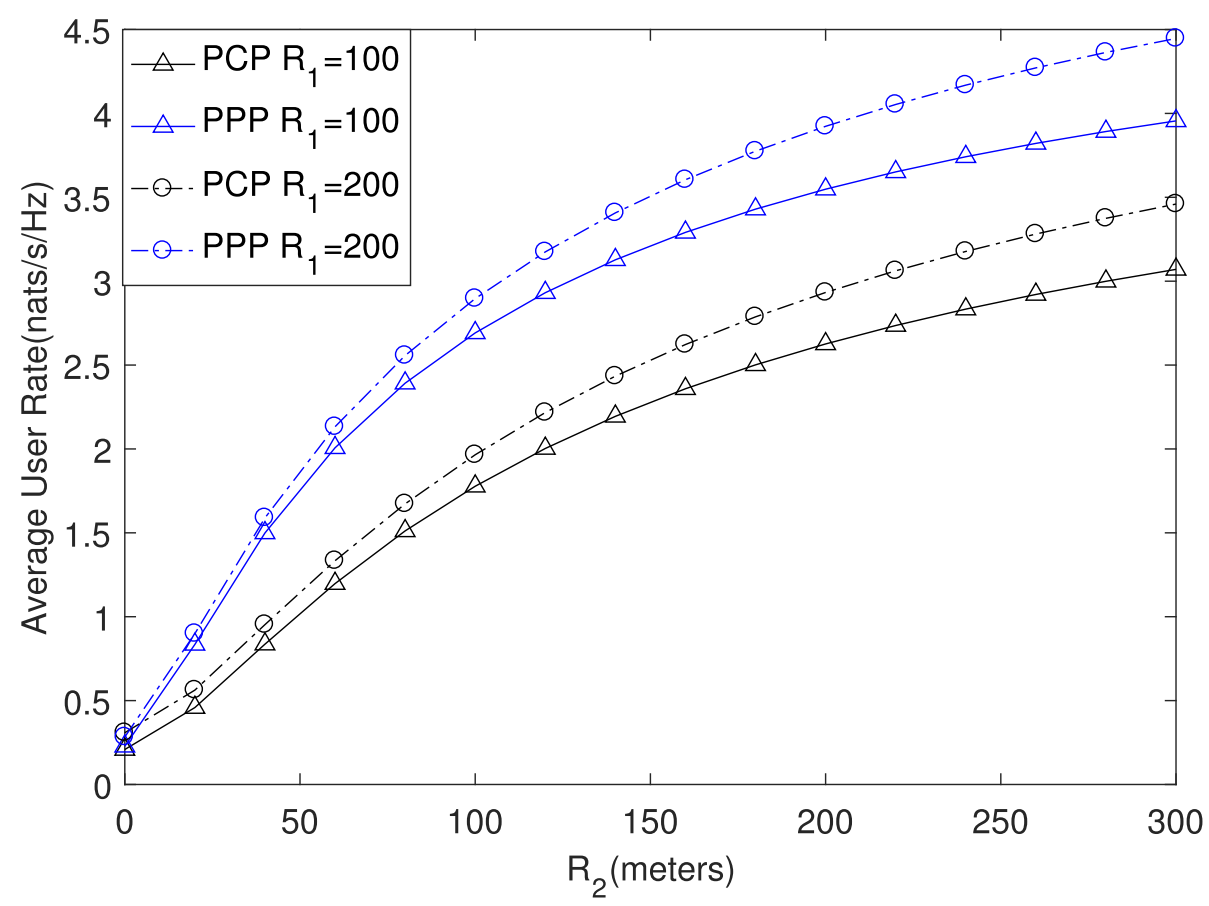

Fig. 5 The average user rates of PPP model and PCP model as a function of the second-tier cooperative radius $R_{2}$ with two different cooperative radii of the first tier 


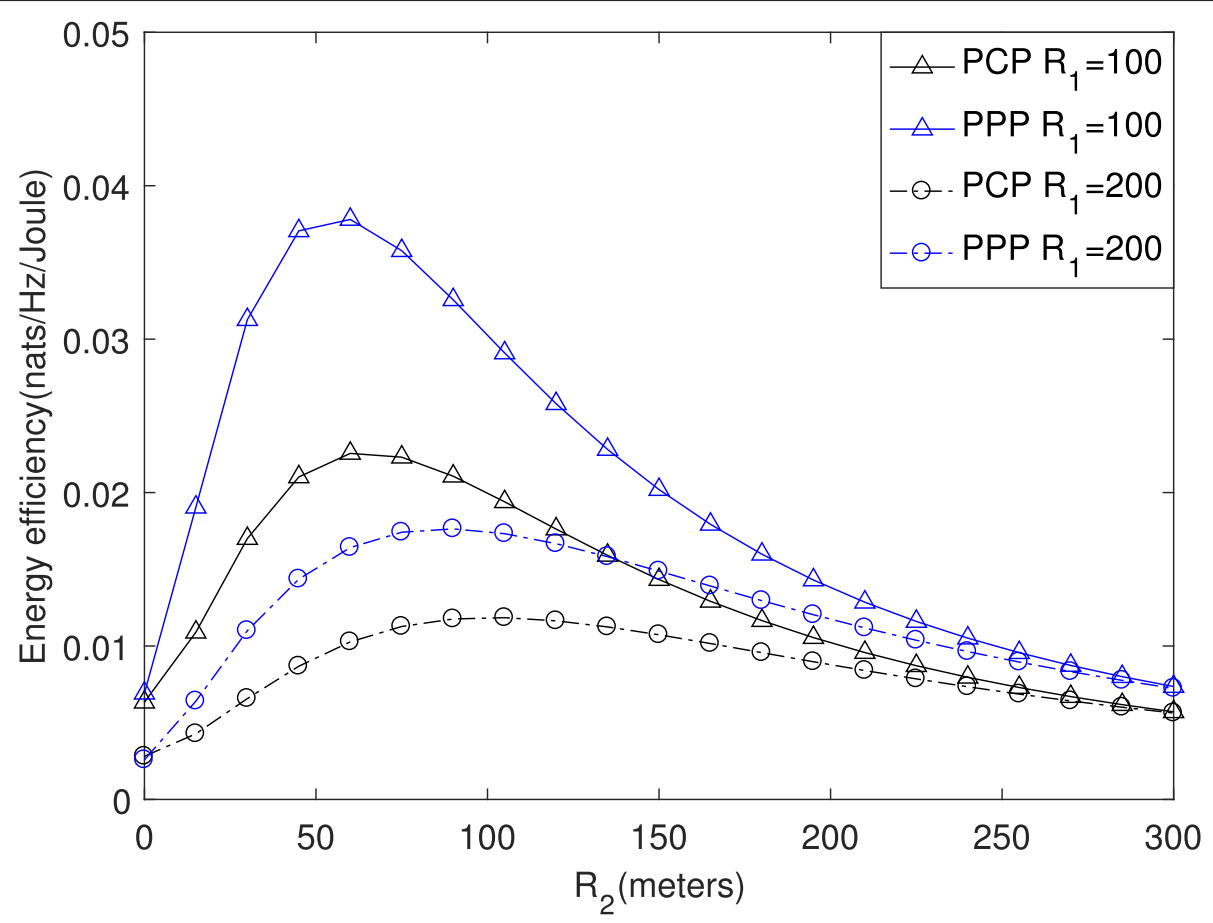

Fig. 6 The energy efficiency of a two-tier network as a function of the first-tier and the second-tier cooperative radius for PPP and PCP

bound curves, and the optimal radius can be calculated approximately by applying the binary search algorithm to (14). It can be seen that the optimal radius increases with the minimum rate $\tau_{0}$, since the increase of the minimum rate $\tau_{0}$ means that more BSs need to be connected to the user, which results in an increase of cooperative radius. Furthermore, the gap between the optimal radius in the case of $R_{1}=300 \mathrm{~m}$ and that in the case of $R_{1}=$ $600 m$ increases with the minimum rate $\tau_{0}$. This is because the macro BSs in a specific cooperative area contribute limited impact on user rate, and more pico BSs are therefore needed to satisfy the increment of the $\tau_{0}$. As $\tau_{0}$ increases, the upper bound increasingly approaches the optimal value, which testifies that the derived closed form is effective, especially for high data rates.

\subsection{Limited number of cooperative BSs and range extension}

Figure 8 shows the average achievable rate versus the second-tier cooperative radius for a different number of cooperative BS based on PPP and PCP. We can see that the achievable rate of PCP is still worse than that of PPP for a fixed number of cooperative BSs, and the reason is the same as for Fig. 5. It can be seen that the achievable rate does not change when $R_{2}$ is greater than a specific value, which reveals that the achieved rate has an upper bound for a given number of cooperative BSs, under either PPP or PCP. The reason is that the number of cooperative $\mathrm{BS}$ reaches the set limit $N$ in the cooperative areas.
Moreover, the increase in the number of associated BSs can improve the achievable rate, but the rate gain increment is a decreasing function of $N$. In the meantime, adding one additional BS means more energy consumption. As a result, BSs located at the edge of cooperative areas is likely to decrease the EE, which also justifies limiting the number of cooperative BSs.

Figure 9 presents the simulation results of the EE based on RE with respect to power bias. Here, we assume that the two-tiers' power biases are both equal to $\varepsilon$. It can be seen that the EE is a concave function of the bias. No matter PCP or PPP, there exists an optimal bias (approximately $\varepsilon=7 \mathrm{~dB}$ ) and it seems to be nearly independent of the normalized RSS threshold. This figure reveals the $\mathrm{RE}$ can improve EE as a consequence of decreasing the macro BS loading in comparison with the non-RE scheme (i.e., $\varepsilon=0 \mathrm{~dB}$ ). Equivalently, it is obvious that the $\mathrm{EE}$ decreases with the normalized RSS threshold $\theta^{*}$ decreasing because of more cluster edge BSs. It is interesting to observe that for $\theta^{*}=-65 \mathrm{dBm}$, the bias $\varepsilon$ has a relatively weaker effect on the EE (due to the large pre-expansion collaboration range). These curves also reveal that the EE of PCP is always lower than that of PPP, as the major interfering BSs in a PCP network are more likely to be closer to the user, which worsens the performances.

\section{Conclusions}

In this paper, we have derived computationally efficient new formulas for Laplace transforms of the aggregate 


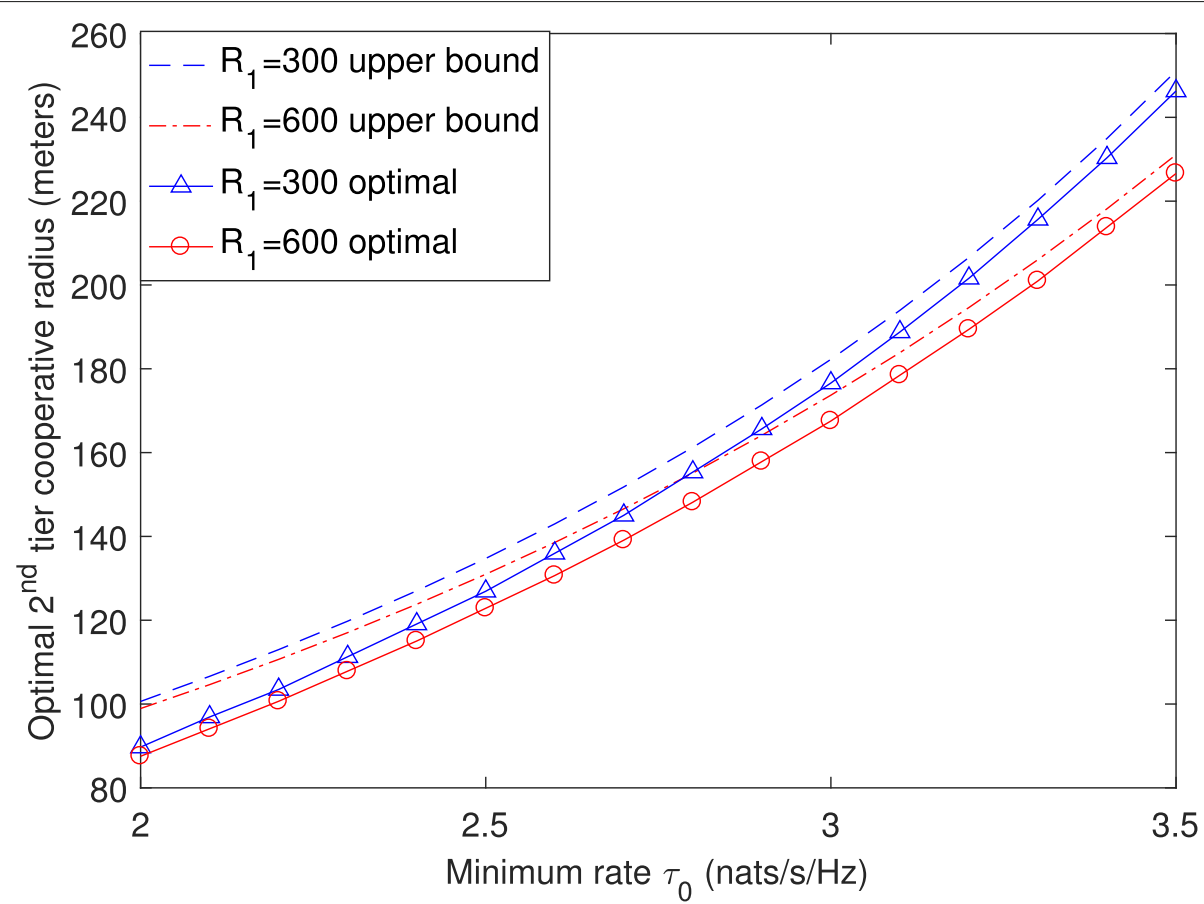

Fig. 7 The optimal cooperative radius as a function of the minimum rate with two different cooperative radii of the first tier

interference and total signal power for PCP HetNets. By using these formulas, we have derived the spectral efficiency and the energy efficiency expressions for a typical user. We have demonstrated that the performances of PCP networks are worse than those of PPP networks.
The optimal cooperative radii are also determined under the condition of a minimum rate, which minimizes the energy consumption. Additionally, we examined the case of limited number of cooperative BSs and the RE scheme (to balance the load between macro BSs and pico BSs).

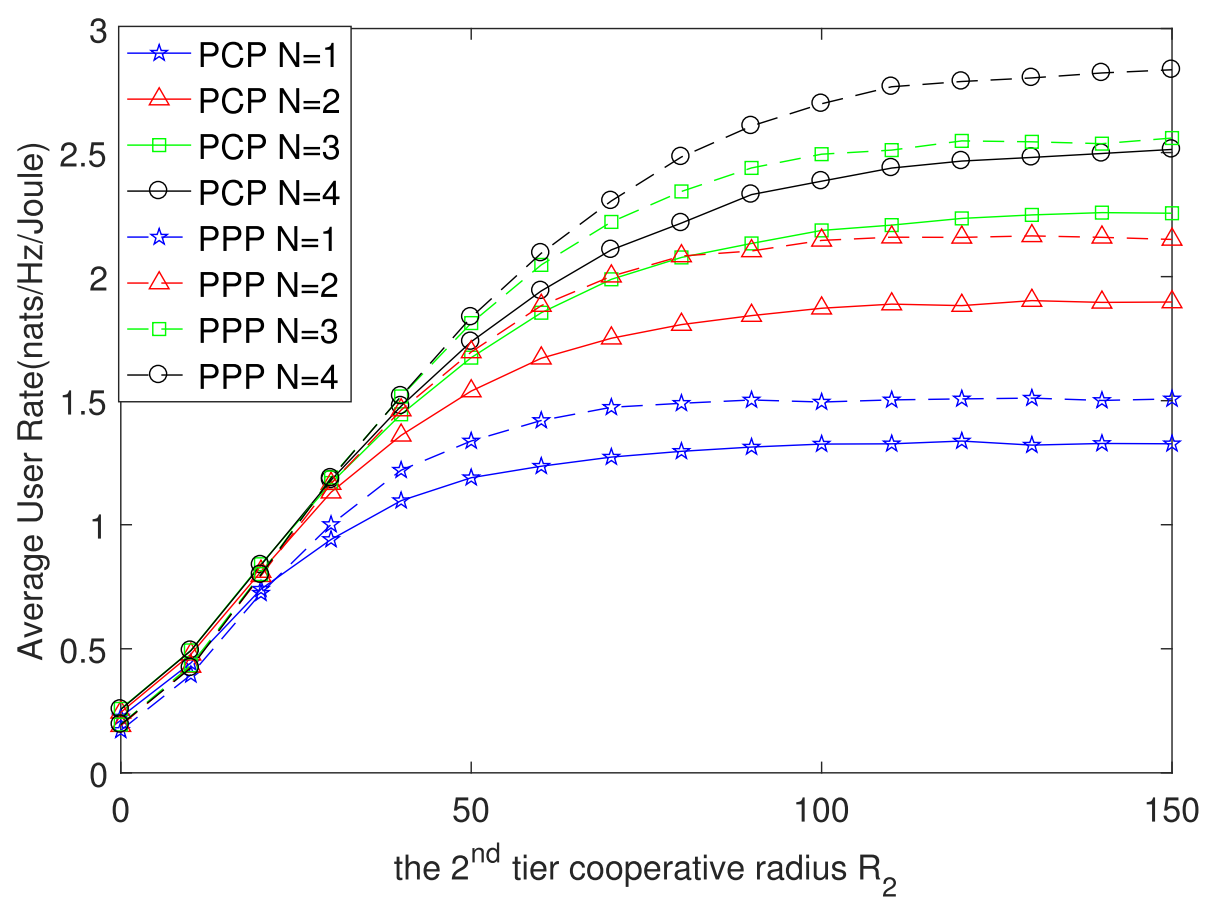

Fig. 8 The average achievable rates as a function of the number of cooperative BSs $(N=1,2,3,4)$ 


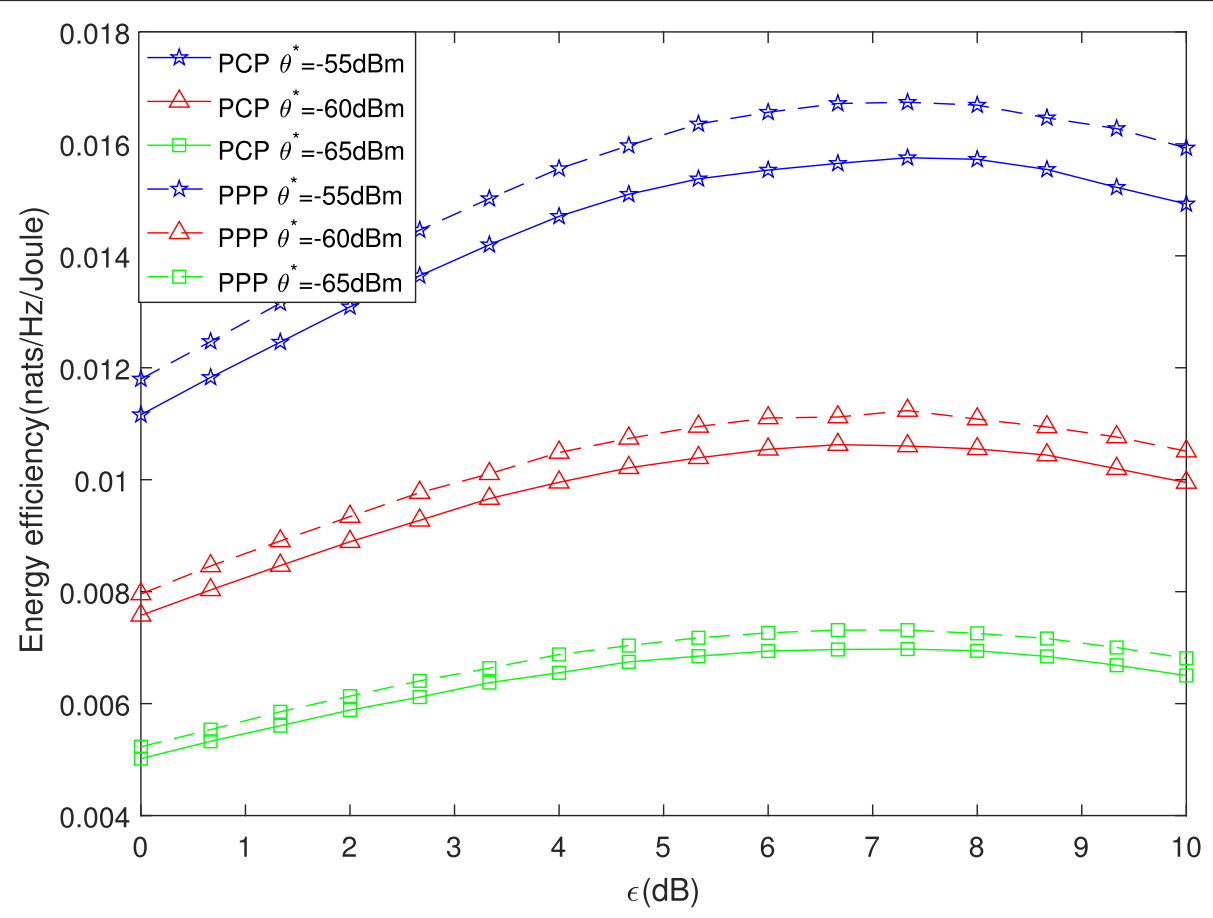

Fig. 9 The simulation results of the energy efficiency based on RE with the cooperative bias factors $\varepsilon$

In terms of energy efficiency, we observed that there exists an optimal solution for the bias, which maximizes the energy efficiency.

\section{Appendix 1. Proof of Theorem 1}

From [27], we have the following useful lemma

$$
\begin{aligned}
\ln (1+x) & =\int_{0}^{\infty} \frac{1}{t}\left(1-e^{-x t}\right) e^{-t} d t \\
& =\int_{0}^{\infty} \frac{1}{t}\left(e^{-t}-e^{-(x+1) t}\right) d t \quad \forall x>0 .
\end{aligned}
$$

By applying (22) to (9), the rate is given by

$$
\begin{aligned}
\tau & =E_{P, I}\left[\int_{0}^{\infty} \frac{1}{t}\left[\exp (-t)-\exp \left(-\frac{P+\sigma^{2}}{I+\sigma^{2}} t\right)\right] d t\right] \\
\stackrel{(a)}{=} & E_{P, I}\left[\int_{0}^{\infty} \frac{e^{-s \sigma^{2}}}{s}[\exp (-s I)-\exp (-s P)] d s\right] \\
& =\int_{0}^{\infty} \frac{e^{-s \sigma^{2}}}{s}\left[\mathcal{L}_{I}(s)-\mathcal{L}_{P}(s)\right] d s
\end{aligned}
$$

where (a) follows by substituting $t=s\left(I+\sigma^{2}\right)$, and $\mathcal{L}_{I}(s)$ denotes the Laplace transform of the aggregate interference power of HetNets.

The group of interfering BSs is denoted by $C_{I}=\Phi \backslash C_{C}$, and the aggregate interference range is outside the circle with radius $R_{k}$. Therefore, following a similar process to Lemma 1, we can express approximately the Laplace transform of aggregate interference as

$$
\begin{aligned}
\mathcal{L}_{I}(s) & =E_{I}(\exp (-s I)) \\
& \stackrel{(a)}{\approx} \prod_{k=1}^{K} \exp \left\{\lambda_{p, k} 2 \pi \int_{R_{k}}^{\infty}\left[B_{k}(y)^{c_{k}}-1\right] y d y\right\},
\end{aligned}
$$

where (a) follows from the fact that BS position can be approximated by the relevant cluster center.

Finally, by substituting (7) and (24) into (23), we can obtain (10) in Theorem 1.

\section{Appendix 2. Proof of Lemma 2}

Proof A useful result from [28] is given by

$$
\int_{0}^{\infty}\left[\exp \left(-v x^{p}\right)-\exp \left(-\mu x^{p}\right)\right] \frac{d x}{x}=\frac{1}{p} \ln \frac{\mu}{v}
$$

where a real part of $\mu$ and $v$ is greater than zero.

The first integral in (10) is 


$$
\begin{aligned}
& \int_{R_{k}}^{\infty}\left[1-\left(1+s p_{k}\|y\|^{-\alpha_{k}}\right)^{-c_{k}}\right] y d y \\
& \stackrel{(a)}{=} \frac{\left(s p_{k}\right)^{\frac{2}{\alpha_{k}}}}{2} \int_{\left(s p_{k}\right)^{-\frac{2}{\alpha_{k}}} R_{k}^{2}}^{\infty}\left[1-\left(1+u^{-\frac{\alpha_{k}}{2}}\right)^{-c_{k}}\right] d u \\
& \stackrel{(b)}{\leq} \frac{\left(s p_{k}\right)^{\frac{2}{\alpha_{k}}}}{2} \int_{\left(s p_{k}\right)^{-\frac{2}{\alpha_{k}}} R_{k}{ }^{2}}^{\infty} c_{k} u^{-\frac{\alpha_{k}}{2}} d u \\
& =\frac{c_{k}}{\alpha_{k}-2} p_{k} R_{k}^{2-\alpha_{k}} s,
\end{aligned}
$$

where (a) is from substituting $\|u\|=\left(s p_{k}\right)^{-\frac{2}{\alpha_{k}}}\|y\|^{2}$, and (b) follows from the fact that $1-(1+x)^{-n} \leq n x$.

Then, by substituting (26) into (10), we ignore the noise and focus on the interference, and the spectral efficiency is approximately calculated as

$$
\begin{aligned}
\tau & \geq \int_{0}^{\infty}\left[\exp \left\{-\sum_{k=1}^{K} \lambda_{p, k} \pi \frac{2 c_{k}}{\alpha_{k}-2} p_{k} R_{k}^{2-\alpha_{k}} s\right\}-\right. \\
& \left.\prod_{k=1}^{K} \exp \left\{-\lambda_{p, k} 2 \pi \int_{0}^{\infty}\left[1-B_{k}(y)^{c_{k}}\right] y d y\right\}\right] \frac{1}{s} d s \\
& =\int_{0}^{\infty} \frac{1}{s}[\exp (-f s)-\exp (-s)] d s+q \\
& \stackrel{(a)}{=} \ln \frac{1}{f}+q=q-\ln f,
\end{aligned}
$$

where (a) follows from fact that $v=f, \mu=1$ and $p=1$ in (25).

\section{Appendix 3. Proof of Theorem 2}

By applying the Lagrangian multiplier method [29], we can establish the objective function as follows:

$$
\min _{\left\{R_{1}, R_{2}, \cdots, R_{K}\right\}} F=P_{u 1}+\zeta\left(q-\ln f-\tau_{0}\right)
$$

Differentiating (28) with respect to $R_{1}, R_{2}, \cdots, R_{K}$, and $\zeta$, respectively, we can obtain

$$
\begin{aligned}
& \frac{\partial F}{\partial R_{1}}=\frac{P_{a, 1}}{p_{1}} L_{1}-\frac{\zeta L_{1}}{\exp \left(q-\tau_{0}\right)} R_{1}^{-\alpha_{1}}=0 \\
& \frac{\partial F}{\partial R_{2}}=\frac{P_{a, 2}}{p_{2}} L_{2}-\frac{\zeta L_{2}}{\exp \left(q-\tau_{0}\right)} R_{2}^{-\alpha_{2}}=0 \\
& \cdots \cdots \\
& \frac{\partial F}{\partial R_{i}}=\frac{P_{a, i}}{p_{i}} L_{i}-\frac{\zeta L_{i}}{\exp \left(q-\tau_{0}\right)} R_{i}^{-\alpha_{i}}=0 \\
& \cdots \ldots \\
& \frac{\partial F}{\partial \zeta}=q-\ln f-\tau_{0}=0
\end{aligned}
$$

where $L_{i}=2 \pi \lambda_{p, i} c_{i} p_{i}$.

Obviously, we can select one equation arbitrarily from (29) to determine the coefficient $\zeta$ :

$$
\zeta=f \frac{P_{a, i}}{p_{i}} R_{i}^{\alpha_{i}}
$$

$R_{o, 1}, R_{o, 2}, \cdots, R_{o, k}, \cdots, R_{o, K}$ satisfy these equations. Then, we can obtain (16) and (17) in Theorem 2.

\begin{abstract}
Abbreviations
BS: Base station; CoMP: Coordinated multi-point; RSS: Received signal strength; EE: Energy efficiency; HetNets: Heterogeneous cellular networks; KPI: Key performance indicator; MCP: Matern cluster process; MIMO: Multiple-input multiple-output; PCP: Poisson cluster process; PGFL: Probability generating functional; PPP: Poisson point process; RE: Range expansion; SE: Spectral efficiency; SINR: Signal-to-interference-plus-noise ratio
\end{abstract}

\section{Authors' contributions}

F-CZ proposed the main ideas and directions, supervised the research, and modified and rewrote some sections of the paper. XJ performed simulations and wrote a draft of the paper. Both authors read and approved the final manuscript.

\section{Funding}

This work was supported in part by a Shenzhen city/HITSZ start-up grant entitled "Energy-Efficient Low-Latency Wireless Networks," the Shenzhen Science and Technology Innovation Commission under Grant JCYJ20180306171815699, the National Basic Research Program of China (973 Program) under grant 2012CB316004, and the UK Engineering and Physical Sciences Research Council under Grant EP/K040685/2.

\section{Availability of data and materials}

Data sharing is not applicable to this article as no datasets were generated or analyzed during the current study.

\section{Competing interests}

The authors declare that they have no competing interests.

Received: 30 July 2018 Accepted: 11 September 2019

Published online: 24 October 2019

\section{References}

1. M. Kamel, W. Hamouda, A. Youssef, Ultra-Dense Networks: A Survey, vol. 18, (2016), pp. 2522-2545

2. Y. Jiang, Q. Liu, F. Zheng, X. Gao, X. You, Energy-efficient joint resource allocation and power control for D2D communications. IEEE Trans. Veh. Technol. 65(8), 6119-6127 (2016)

3. T. Zhang, J. Zhao, L. An, D. Liu, Energy efficiency of base station deployment in ultra dense HetNets: A stochastic geometry analysis. IEEE Wirel. Commun Lett. 5(2), 184-187 (2016). https://doi.org/10.1109/LWC.2016.2516010

4. S. Khan, S. A. Mahmud, in IEEE International Conference on Computer and Information Technology; Pervasive Intelligence and Computing, Liverpool. Power Optimization Technique in interference-limited femtocells in LTE and LTE advanced based femtocell networks, (2015), pp. 749-754. https:// doi.org/10.1109/cit/iucc/dasc/picom.2015.110

5. S. Geirhofer, P. Gaal, in 2012 IEEE Globecom Workshops. Coordinated multi point transmission in 3GPP LTE heterogeneous networks, (Anaheim, 2012), pp. 608-612. https://doi.org/10.1109/glocomw.2012.6477643

6. R. Irmer, et al., Coordinated multipoint: Concepts, performance, and field trial results. IEEE Commun. Mag. 49(2), 102-111 (2011)

7. A. He, D. Liu, Y. Chen, T. Zhang, in Annual International Symposium on Personal, Indoor, and Mobile Radio Communication (PIMRC). Stochastic geometry analysis of energy efficiency in HetNets with combined CoMP and BS sleeping, (Washington DC, 2014), pp. 1798-1802. https://doi.org/ 10.1109/pimrc.2014.7136461

8. J. G. Andrews, F. Baccelli, R. K. Ganti, A tractable approach to coverage and rate in cellular networks. IEEE Trans. Commun. 59(11), 3122-3134 (2011)

9. R. W. Heath, M. Kountouris, T. Bai, Modeling heterogeneous network interference using Poisson point processes. IEEE Trans. Signal Proc. 61(16), 4114-4126 (2013)

10. W. Nie, F. C. Zheng, X. Wang, W. Zhang, S. Jin, User-centric cross-tier base station clustering and cooperation in heterogeneous networks: Rate improvement and energy saving. IEEE J. Sel. Areas Commun. 34(5), 1192-1206 (2016) 
11. V. Suryaprakash, J. M $\phi \| l e r, G$. Fettweis, On the modeling and analysis of heterogeneous radio access networks using a Poisson cluster process. IEEE Trans. Wirel. Commun. 14(2), 1035-1047 (2015)

12. Q. Ying, Z. Zhao, Y. Zhou, R. Li, X. Zhou, H. Zhang, Characterizing spatial patterns of base stations in cellular networks, (Shanghai, 2014), pp. 490-495 https://doi.org/10.1109/iccchina.2014.7008327

13. C.-H. Lee, C.-Y. Shih, Y.-S. Chen, Stochastic geometry based models for modeling cellular networks in urban areas. Wirel. Netw. 19(6), 1063-1072 (2013)

14. C. Kong, et al., VSMC MIMO: A spectral efficient scheme for cooperative relay in cognitive radio networks, (2015), pp. 2137-2145. https://doi.org/10.1109/ infocom.2015.7218599

15. M. Demirtas, A. Soysal, in IEEE 81st Vehicular Technology Conference (VTC Spring). Energy and spectral efficient microcell deployment in heterogeneous cellular networks, (2015), pp. 1-5. https://doi.org/10.1109/ vtcspring.2015.7145803

16. Y. Lin, W. Yu, Ergodic capacity analysis of downlink distributed antenna systems using stochastic geometry. Proc. IEEE Int. Conf. Commun. (ICC), 3338-3343 (2013). https://doi.org/10.1109/icc.2013.6655062

17. J. Zhao, Q. Wang, Y. Dong, W. Wei, in IEEE Wireless Communications and Networking Conference. Performance analysis for cross-tier cooperation in heterogeneous cellular networks: A stochastic geometry approach, (Doha, 2016), pp. 1-6. https://doi.org/10.1109/wenc.2016.7564964

18. W. Yi, Y. Liu, A. Nallanathan, Modeling and Analysis of D2D MillimeterWave Networks With Poisson Cluster Processes. IEEE Trans. Commun. 65(12), 5574-5588 (2017). https://doi.org/10.1109/TCOMM.2017.2744644

19. Y. J. Chun, M. O. Hasna, A. Ghrayeb, Modeling heterogeneous cellular networks interference using Poisson cluster processes. IEEE J. Sel. Areas Commun. 33(10), 2182-2195 (2015)

20. Y. Wang, Q. Zhu, Modeling and analysis of small cells based on clustered stochastic geometry. IEEE Commun. Lett. 21(3), 576-579 (2017)

21. S. N. Chiu, D. Stoyan, W. S. Kendall, J. Mecke, Stochastic Geometry and Its Applications, 3rd ed. (Wiley, Hoboken, 2013)

22. R. K. Ganti, M. Haenggi, Interference and outage in clustered wireless ad hoc networks. IEEE Trans. Inf. Theory. 55(9), 4067-4086 (2009)

23. L. Zhang, S. Zhao, P. Shang, J. Liu, F. Han, in IEEE 85th Vehicular Technology Conference (VTC Spring). Distributed adaptive range extension setting for small cells in heterogeneous cellular network, (Sydney, NSW, 2017), pp. 1-7. https://doi.org/10.1109/vtcspring.2017.8108474

24. Q. Ye, B. Rong, Y. Chen, M. Al-Shalash, C. Caramanis, J. G. Andrews, User association for load balancing in heterogeneous cellular networks. IEEE Trans. Wirel. Commun. 12(6), 2706-2716 (2013)

25. H. S. Jo, Y. J. Sang, P. Xia, J. G. Andrews, Heterogeneous cellular networks with flexible cell association: A comprehensive downlink SINR analysis. IEEE Trans. Wirel. Commun. 11(10), 3484-3495 (2012)

26. G. Auer, et al., How much energy is needed to run a wireless network? IEEE Wirel. Commun. Mag. 18(5), 40-49 (2011)

27. K. A. Hamdi, Capacity of MRC on correlated Rician fading channels. IEEE Trans. Commun. 56, 708-711 (2008)

28. A. Jeffrey, D. Zwillinger, Table of Integrals, Series, and Products. (Academic, New York, 2007)

29. S. Boyd, L. Vandenberghe, Convex Optimization. (Cambridge Univ Press, Cambridge, 2004)

\section{Publisher's Note}

Springer Nature remains neutral with regard to jurisdictional claims in published maps and institutional affiliations.

\section{Submit your manuscript to a SpringerOpen ${ }^{\circ}$ journal and benefit from:}

- Convenient online submission

Rigorous peer review

- Open access: articles freely available online

- High visibility within the field

- Retaining the copyright to your article

Submit your next manuscript at $>$ springeropen.com 